\title{
Electrocorticogram in Rats Loaded with SART Stress (Repeated Cold Stress)
}

\author{
Taeko HATA, Yoshitaka NISHIMURA, Tomitaro KITA, \\ Atsufumi KAWABATA and Eiji ITOH \\ Department of Pharmacology, Faculty of Pharmacy. Kinki University. \\ Kowakae. Higashi-Osaka 577, Japan \\ Accepted July 29, 1987
}

\begin{abstract}
The electrocorticogram (ECOG) in a SART (specific alternation of rhythm in temperature)-stressed (repeatedly cold-stressed) rat, which is regarded as an experimental model for clinical vagotonic-type dysautonomia, was investigated in the present study by the power spectral technique. 1) Analysis of ECoG in SART-stressed rats during the resting-arousal state indicated a decrease in total power and a decrease in relative power in the $\delta$ band, and also an increase in relative power in the $\theta, \alpha$ and $\beta$ bands. 2) In the slow-wave sleeping state, the ECOG of SART-stressed rats indicated a marked increase in total power, an increase in the $\delta$ band and decreases in $\theta, \alpha$ and $\beta$ bands. 3) Electric stimulation of the posteriorhypothalamic area evoked alterations of ECOG similar to those caused by SART stress. ECOG response to electric stimulation in SART-stressed rats was less than that in unstressed rats. 4) Lesioning of the posterior-hypothalamic areas prevented SART stress-induced ECOG alterations. SART-stressed rats thus appear to be at a higher consciousness level on awakening but to sleep more soundly. They seem to exhibit greater fluctuation in brain activity than normal rats. There is also the possibility that the posterior-hypothalamic area is responsible to some degree for ECoG alterations in SART-stressed rats.
\end{abstract}

Numerous reports indicate that experimental animals such as mice, rats and guinea pigs exposed to SART (specific alternation of rhythm in temperature) stress (repeated cold stress) $(1,2)$, a type of subacute stress, come to show various physiological abnormalities in the periphery (3-9). With respect to the central nervous system (CNS), such rats show certain biochemical changes including decreased content of total acetylcholine and increased activity of related enzymes $(10,11)$ as well as increased contents of dopamine and norepinephrine in the brain $(12,13)$. Little, however, is known about the functional states in the CNS.

A SART-stressed animal may be regarded as an experimental model for clinical vagotonic-type dysautonomia, considering the results of the GSR (galvanic skin response), Ashner's occulocardiac and mecholyl tests $(1,14)$. The hearts of such animals are sympathicotonic (6). This complicated imbalance in the autonomic nervous system may be involved in the modulating mechanisms in the CNS.

In the present study, we examined the electrocorticogram (ECOG) of SART-stressed rats by the power spectral technique. Moreover, electric stimulation and lesions of the posterior-hypothalamic area were performed so as to gain some understanding of the mechanism of ECOG alterations produced by SART stress.

\section{Materials and Methods}

Animals and stress procedure: Male adult Wistar rats weighing between 270 and $310 \mathrm{~g}$ were used. For loading SART stress, rats were alternately kept at $24^{\circ} \mathrm{C}$ and $-3^{\circ} \mathrm{C}$ for $1 \mathrm{hr}$-periods from $9 \mathrm{a} . \mathrm{m}$. to $4 \mathrm{p} . \mathrm{m}$. and then at $-3^{\circ} \mathrm{C}$ from 4 p.m. to 9 a.m. the following morning. This was continued for 5 con- 
secutive days $(1,2)$, and stopped on the 6 th morning. The stressed rats were submitted to experiments at least $1 \mathrm{hr}$ after the cessation of stress.

ECoG recording: Under pentobarbital- $\mathrm{Na}$ (40 $\mathrm{mg} / \mathrm{kg}$, i.p.) anesthesia, the rats were made stationary by a stereotaxic apparatus (Narishige, SR-5). Stainless steel screw ulectrodes $(1.2 \mathrm{~mm}$ in diameter) were implanted on the cortical surface through the skull. Electrode placements were the left frontal $(2 \mathrm{~mm}$ anterior and $1.5 \mathrm{~mm}$ lateral to bregma) and bilateral parietal $(3.5 \mathrm{~mm}$ posterior and $2 \mathrm{~mm}$ lateral to bregma) areas. The reference electrode was placed on the intraparietal bone $(1.5 \mathrm{~mm}$ posterior to lamda). All electrodes were fixed in place with acrylic dental cement (Nissin Dental Products Inc., Mend-Rex ${ }^{(\widehat{P})}$. Ten days after the surgery, ECOG was monopolarly recorded in both unanesthetized and unrestrained states during resting-arousal and slow-wave sleep. The electrodes were connected to a polygraph system (Nihon Kohden, RM-6000) through a mercury pool swivel which permitted the animal to move freely without entangling the electrode leads. ECOG was drawn on an ink-writing recorder at a paper speed of $25 \mathrm{~mm} / \mathrm{sec}$ for about $10 \mathrm{~min}$ during monitoring for $60 \mathrm{~min}$ and simultaneously recorded on magnetic tapes (Sony. SIT-90F) using a cassete data recorder (Nihon Kohden, RMG-5204) for later ECoG analysis. The time constant and high-cut filter were set at $0.3 \mathrm{sec}$ and $100 \mathrm{~Hz}$, respectively.

Electric stimulation and lesions of the posterior-hypothalamic area: The posteriorhypothalamic area was defined according to stereotaxic coordinates derived from the atlas of Paxinos and Watson $13.8 \mathrm{~mm}$ posterior and $8.0 \mathrm{~mm}$ ventral to bregma and $0.5 \mathrm{~mm}$ lateral to the midline) (15). A pair of twisted stainless steel wires with epoxyresin coating $(0.2 \mathrm{~mm}$ in diameter and about $0.2 \mathrm{~mm}$ in interpolar distance) served as the electric stimulation electrodes. For the electric stimulation experiments, square wave pulses (a duration of $0.1 \mathrm{msec}, 3$ or $5 \mathrm{~V}$ ) were sent to the left posterior-hypothalamic area at a rate of 100 pulses per second for $15 \mathrm{sec}$ using an electric stimulator (Nihon Kohden.
SEN-3201) and an isolator (Nihon Kohden, SS-102J). ECoG of the left frontal cortex was registered continuously during the stimulation. For the lesion experiments, radiofrequency destruction of the posteriorhypothalamic areas was bilaterally achieved at $65^{\circ} \mathrm{C}$ for $3 \mathrm{~min}$ using a lesion generator (Radionics, model RFG-4A) and an element of $0.25 \mathrm{~mm}$ in diameter (Radionics, type TC). ECoG of the left frontal cortex was recorded.

Implanting of stimulation electrodes and radiofrequency destruction were performed 10 days before stress loading. After the end of the experiments, the brain was isolated and electrode placements and lesions were visually verified.

ECoG analysis: Consecutive $2.56 \mathrm{sec}$ ECOG epochs stored on magnetic tapes were digitized at a rate of $10 \mathrm{msec}$, and then power spectra were obtained by Fast Fourier Transformations, using a data analyzer system (Nihon Kohden. ATAC-450). The sampling time was $10.24 \mathrm{sec}$ in restingarousal states and $20.48 \mathrm{sec}$ in slow-wave sleeping states. The analytical time of 10.24 sec in the resting-arousal ECoG was chosen to obtain stable ECoG without meaningless alterations and to avoid movement artifacts, since rats possess relatively fast sleepawakening cycles during the daytime and move more actively as compared with rabbits and cats $(16,17)$. In the slow-wave sleeping state, as the stable ECoG recordings can be obtained easily, 20.48 sec ECoG samples were analyzed to improve the precision. Subsequently, to quantify the power spectra obtained, total potency and relative power were calculated according to the method described by Young et al. (18) and Wauquier et al. (19), with minor modification. Total power was obtained by adding the powers from $1.56 \mathrm{~Hz}$ to $29.64 \mathrm{~Hz}$, and total potency was expressed as the percentage of the control total power for each animal. The relative power in $\delta(1.56-3.90$ $\mathrm{Hz}) . \theta(3.90-7.80 \mathrm{~Hz}), \alpha(7.80-13.26 \mathrm{~Hz})$ and $\beta(13.26-29.64 \mathrm{~Hz})$ bands was represented as the percentage of total power. After observing the paper ECoG records, several artifact-free epochs were selected and analyzed. The average of these data was taken as the analytical value for each animal. 
Statistical analysis: All data obtained were presented as the mean \pm S.E. Significant differences were analyzed by Student's $t$-test.

\section{Results}

Resting-arousal ECoG in SART-stressed rats: Direct left-frontal ECOG recordings of a rat during the resting-arousal state before and after SART stress and corresponding power spectra are shown in Fig. 1. As seen in the upper part of this figure, a decrease in $\delta$ waves and increase in $\theta$ waves were observed after the stress, and the ECoG exhibited low-amplitude fast waves as a whole. The lower part of Fig. 1 shows the histograms of the power spectra. The peak power shifted from $2-4 \mathrm{~Hz}$ to $5-7 \mathrm{~Hz}$ due to the stress.

In SART-stressed rats, as seen in Fig. 2. total power significantly decreased as compared to normal (before stress) rats, and total potency in the left frontal, left and right parietal areas was 77,81 and $84 \%$. respectively. As to the relative power of SART-stressed rats, the $\delta$ band significantly decreased, and the $\theta$ band significantly increased in all three areas. The relative power of the $\alpha$ and $\beta$ bands tended to increase, although the magnitude of change differed according to the area.

As described above, there was little difference among these three areas in change caused by stress in total potency and relative power. Then, left-frontal ECoG recordings were shown in the following results.

Slow-wave sleep ECoG in SART-stressed rats: Representative left-frontal ECoG recordings before and after SART stress during the slow-wave sleep are shown in Fig. 3. The ECoG after stress was visually observed and found to have higher amplitude and lower frequency than before stress. The histograms of power spectra for $20.48 \mathrm{sec}$ containing these $\mathrm{ECoG}$ samples drawn at
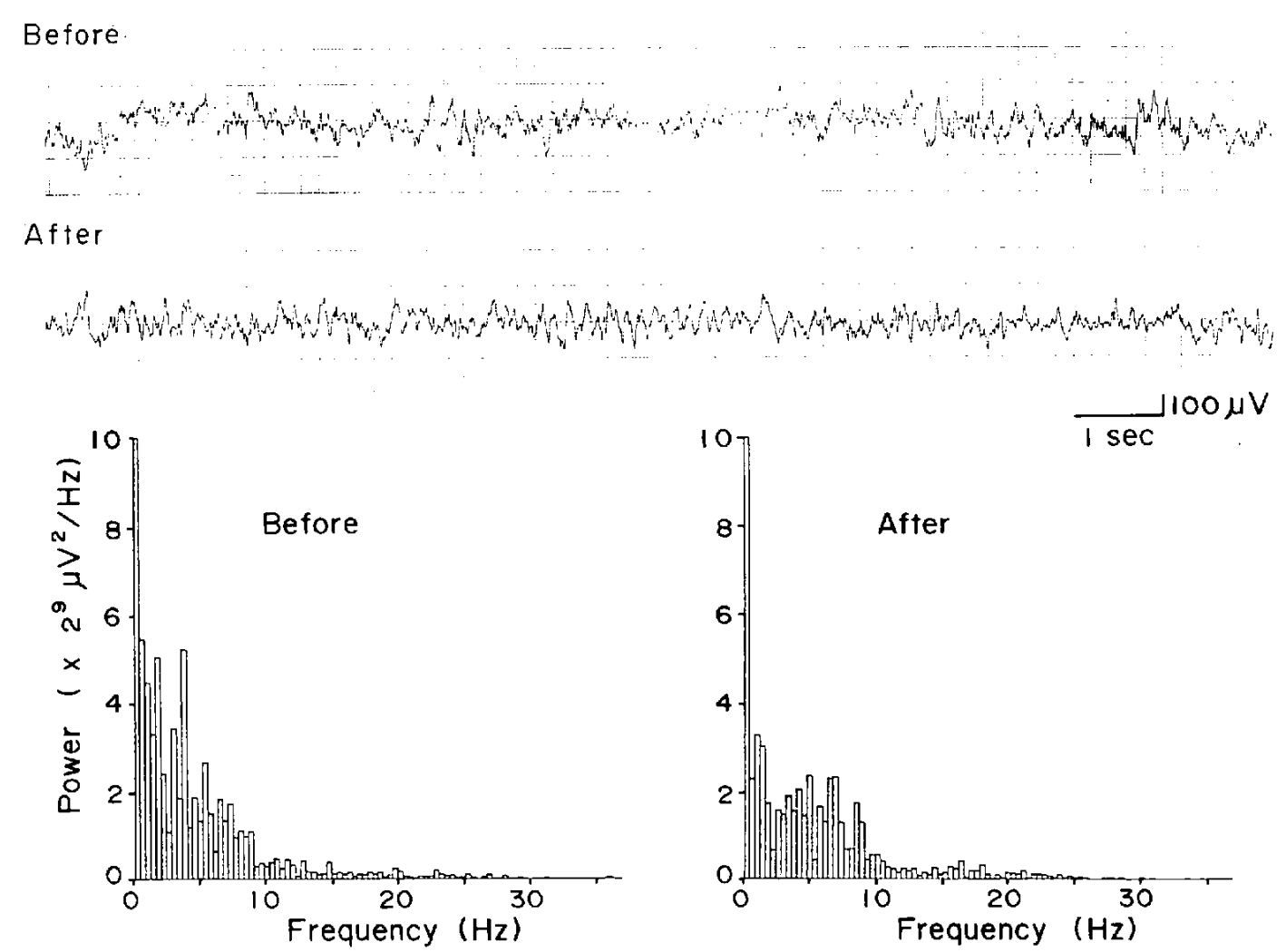

Fig. 1. Direct resting-arousal ECoG recordings and their power spectra at the left frontal area of a rat before and after SART stress. 


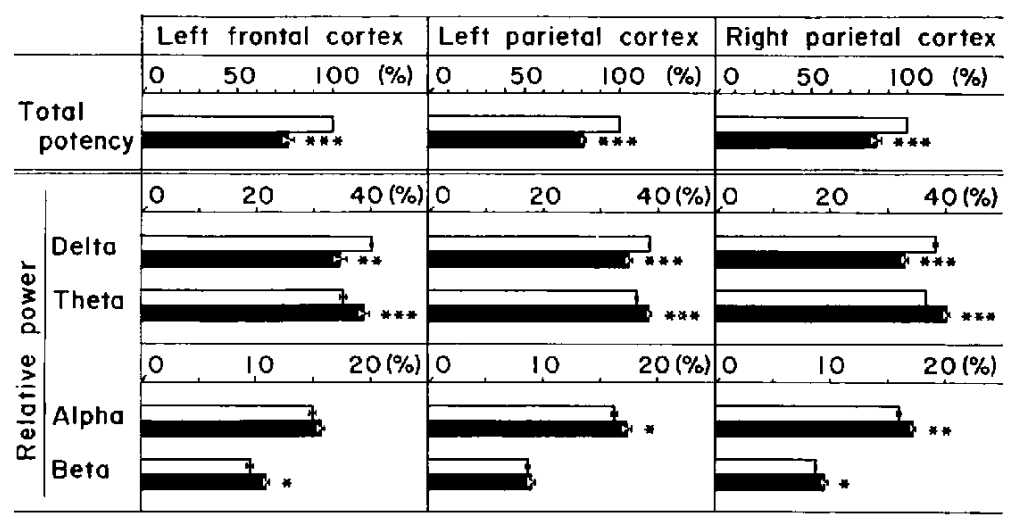

Fig. 2. Power spectral analysis of resting-arousal ECOG in non-stressed and SART-stressed rats. $\square$ : Non-stress, : SART stress. Values are means \pm S.E. of 23-27 non-stressed and 7-10 SART-stressed rats. ${ }^{*} P<0.05,{ }^{* *} P<0.01$ and ${ }^{* *} P<0.001$ (t-test).

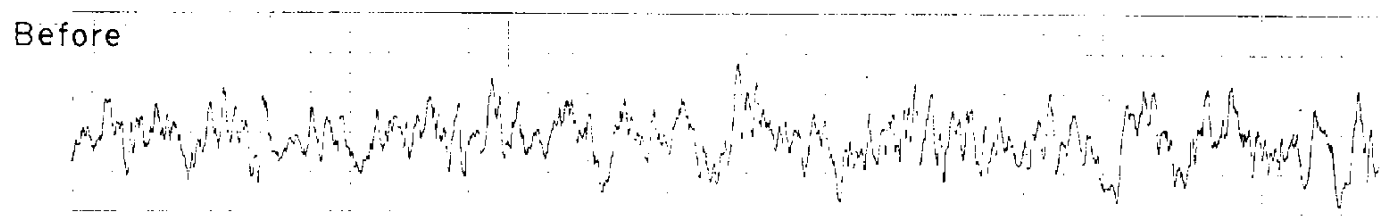

After
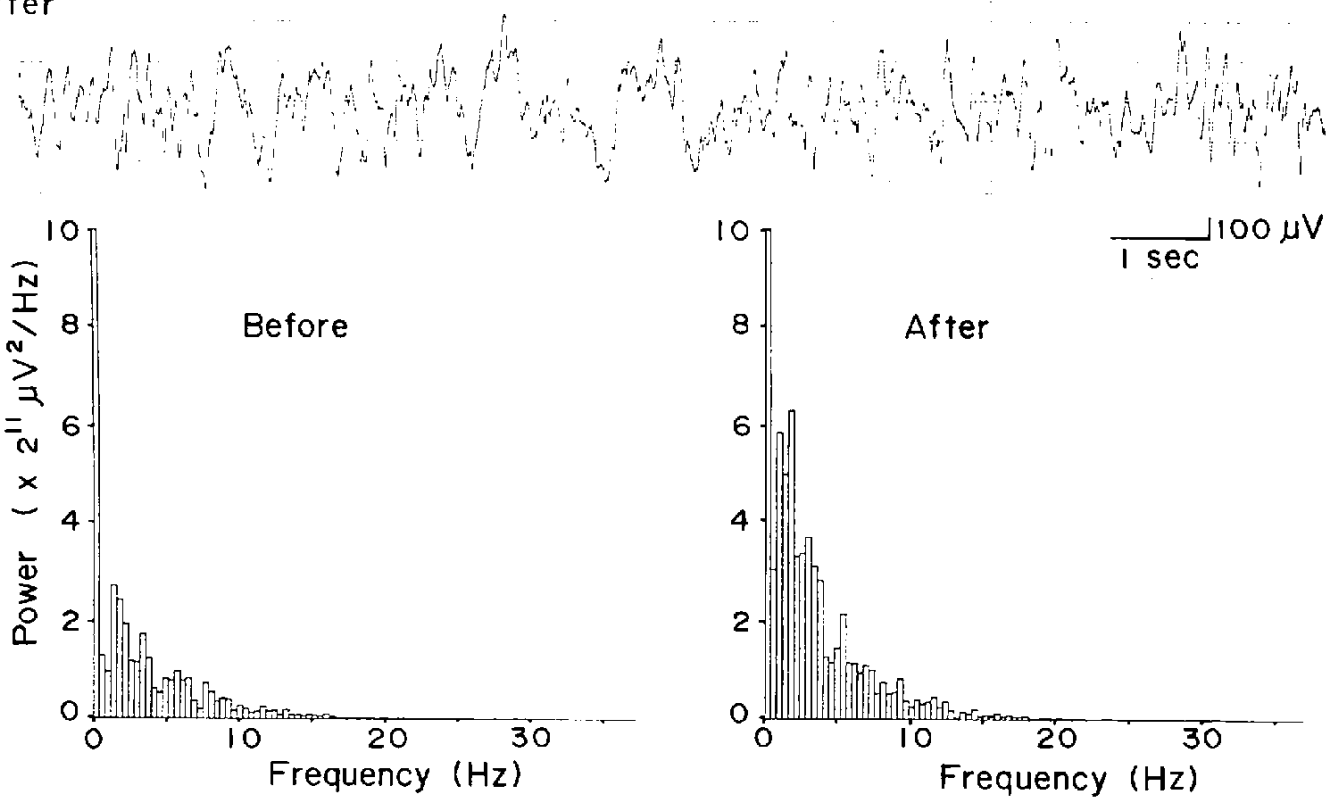

Fig. 3. Direct slow-wave sleep ECOG recordings and their power spectra at the left frontal area of a rat before and after SART stress.

the upper panel are shown in the lower part of Fig. 3. After stress, most of the ECOG spectral power was evident in the $0-5 \mathrm{~Hz}$ band.

The average total potency and relative power of the left-frontal ECOG in slow-wave 
sleep states are shown in Fig. 4. SARTstressed rats indicated a markedly increased total potency of $195 \%$, increased $\delta$ band and decreased $\theta, \alpha$ and $\beta$ bands in relative power.

In Fig. 5, to summarize and characterize ECOG alterations in SART-stressed rats, differences between relative powers in leftfrontal ECoG before and after stress are expressed as $\Delta$ relative power. The ECoG of SART-stressed rats was characterized by faster waves in resting-arousal states and slower waves in slow-wave sleeping states. compared to unstressed, normal rats.

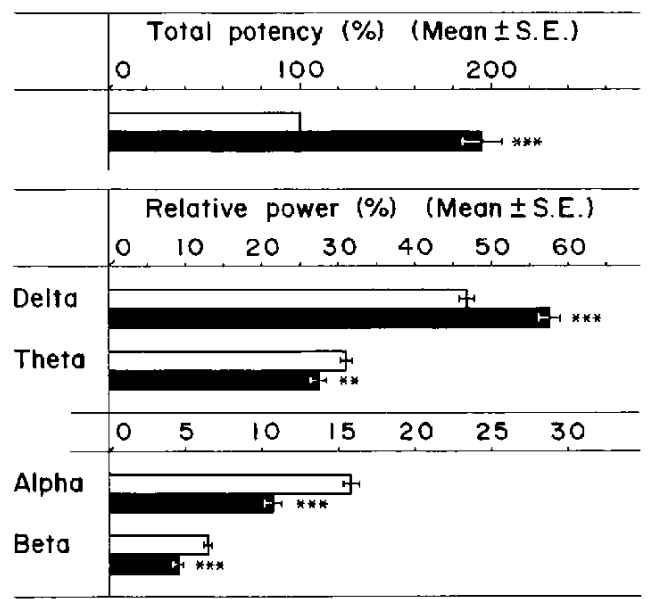

Fig. 4. Power spectral analysis of slow-wave sleep ECOG at the left frontal area in non-stressed and SART-stressed rats. $\square$ : Non-stress, $\square$ : SART stress. No. of rats: 12/group. ${ }^{* *} P<0.01$ and ${ }^{* *} \mathrm{P}<0.001$ ( $t$-test).
The influence of electric stimulation and lesions of the posterior-hypothalamic area on resting-arousal ECoG: Figure 6 shows the results from the power spectra analysis of resting-arousal ECOG in the left frontal area induced by electric stimulation of left posterior-hypothalamic area in non-stressed and SART-stressed rats. In non-stressed rats, the total potency decreased in a voltagedependent manner by stimulation. The relative power of the $\delta$ and $\theta$ bands showed voltage-related decrease and increase, respectively. Also, significant increase in $\alpha$ and $\beta$ bands was noted by a stimulation of $3 \mathrm{~V}$ and/or $5 \mathrm{~V}$. In the SART-stressed rats, a slight decrease in total potency occurred by electric stimulation, but was not significant. The relative power of the $\delta$ and $\theta$ bands showed voltage-related decrease and increase, respectively, but the magnitude of change in SART-stressed rats was less than that in non-stressed rats. Moreover, the ECoG alterations elicited by $3 \mathrm{~V}$-electric stimulation in the non-stressed rats were similar to those caused by SART stress.

The results obtained from power spectral analysis of resting-arousal ECoG in the left frontal area of posterior hypothalamuslesioned rats before and after stress loading are presented in Fig. 7. The bilateral posteriorhypothalamic lesions caused a decrease in the $\delta$ band and a slight increase in the $\theta$ band in non-stressed rats. When the rats were loaded with SART stress after posteriorhypothalamic lesions, no change in total potency and relative power caused by SART

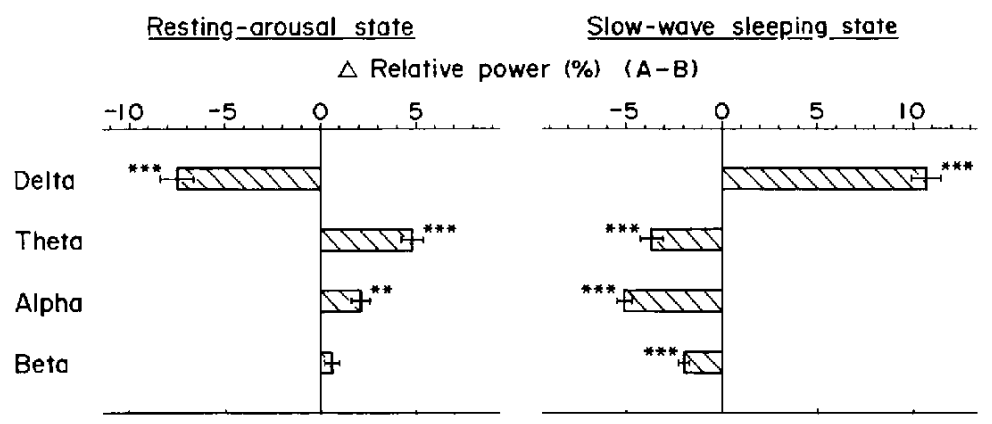

Fig. 5. Changes of rat ECOG at the left frontal cortex caused by SART stress. A: The value after SART stress, B: The value before SART stress. Values are means $\pm S$. E. of $g$ or 12 rats/group. $* * P<0.01$ and ${ }^{* * *} \mathrm{P}<0.001$ ( $t$-test). 


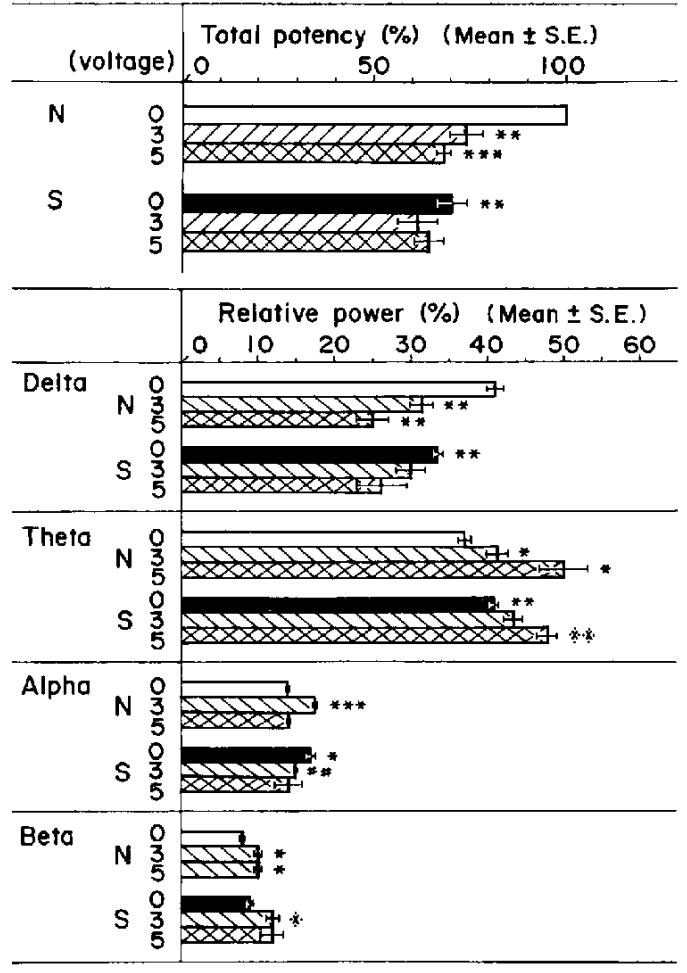

Fig. 6. Influence of electric stimulation at the left posterior-hypothalamic area on resting-arousal ECOG at the left frontal area of rats. N: Non-stress, S: SART stress. No. of rats: 3 or $4 /$ group. ${ }^{*} P<0.05$. ${ }^{*} \mathrm{P}<0.01$ and ${ }^{* *} \mathrm{P}<0.001$, compared to nonstressed control, $\because P<0.05$ and $\% * P<0.01$, compared to SART-stressed control, and $\$ P<0.01$, compared to respective non-stressed group ( $t$-test).

stress could be detected, and the data obtained were essentially the same values as before the stress.

\section{Discussion}

In the present study, the ECOG of SARTstressed rats was investigated by the power spectral technique. Resting-arousal ECoG alterations in SART-stressed rats were expressed as decreased total potency, and decreased $\delta$ band and increased $\theta, \alpha$ and $\beta$ bands in relative power. These results show SART-stressed rats to have low-amplitude fast waves. It has been reported that power spectra are influenced by functional changes in the brain $(20,21)$, various CNS acting drugs $(19,22-24)$ and stress (25). Yamamoto

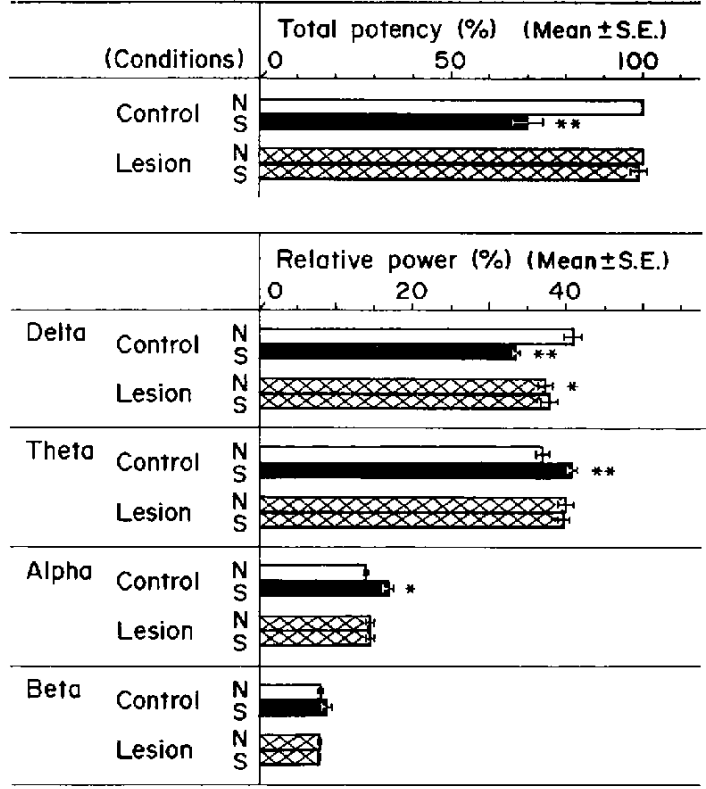

Fig. 7. Influence of bilateral posterior-hypothalamic lesions on resting-arousal ECOG at the left frontal area of rats. N: Non-stress, S: SART stress. Values are means=S.E. of $4-6$ rats/group. ${ }^{*} P<0.05$ and ${ }^{*} P<0.01$, compared to non-stressed control ( $t$ test).

(24) reported that in rabbits, a decrease in total power accompanied by that in peak power occurred with enhancement of the consciousness level. It is evident that SARTstressed rats were at an enhanced consciousness level, since a decrease in total power accompanied by a shift to higher frequency of peak power was noted in SARTstressed rats. In the human EEG, the suppression of $\alpha$ rhythm and dominant fast waves have been observed when the brain activity level is augmented by mental excitement and attention. From the GSR test results (1), SART-stressed rats fall into hypersensitivity by external stimuli. Considering low-amplitude fast waves of restingarousal ECoG in SART-stressed rats, the SART-stressed rats may possibly be in a state of mental tension.

Various types of stress have been reported to evoke changes in the ECoG of experimental animals (25-28). Harada et al. (27) reported that the $\mathrm{ECOG}$ in rats exposed to restraint stress fluctuated between low-voltage 
rhythmical waves and high-voltage irregular waves including spindles and spikes, and that rats restrained in $25^{\circ} \mathrm{C}$ water showed only low-voltage rhythmical waves, the frequency of which increased in the early stages of the stress, gradually decreasing below the levels prior to the stress. According to Iwata and Mikuni (25), the relative activity in the range $7.5-13.0 \mathrm{~Hz}$ markedly increased in rats exposed to fearinduced stress. When rats were kept at -10 to $-15^{\circ} \mathrm{C}$ for $12 \mathrm{hr}$, no change occurred in ECOG (26). ECOG alterations in SART. stressed rats, therefore. markedly differ from those evoked by restraint, fear-inducement and acute cold stress.

Recently, many patients with psychosomatic diseases have been reported to show irregular $\alpha$ waves and fast dominant waves in their EEG (29). Therefore, it is of much interest that low-amplitude fast waves were seen in SART-stressed rat ECoG in this study.

Slow-wave sleep ECoG in SART-stressed rats showed higher-amplitude slower waves as compared with those in normal rats. SARTstressed rats may sleep more soundly than normal rats. REM-sleep ECoG and the sleepawakening cycle in SART-stressed rats remain to be investigated.

It is well-known that the arousal response of EEG is induced by electric stimulation of the posterior hypothalamus. In this study, ECOG alterations evoked by $3 \mathrm{~V}$-stimulation in normal rats were similar to those caused by SART stress. Response to electric stimulation in SART-stressed rats was less than that in normal rats. In contrast, lesioning of the posterior-hypothalamic areas inhibited ECOG alterations caused by SART stress. From these results, the posterior-hypothalamic area appears to be involved at least in ECoG alterations in SART-stressed rats, although it will be necessary to examine brainstem reticular formation and other parts of the brain.

In conclusion, SART-stressed rats may possibly be at a higher consciousness level on awakening and sleep more soundly. There is greater fluctuation in brain activity than in normal rats.

We are currently investigating effects of some drugs on these alterations of ECoG and behavioral characterization in SARTstressed animals.

\section{References}

1 Kita, T., Hata, T., Yoneda, R. and Okage, T.: Stress state caused by alternation of rhythm in environmental temperature, and the functional disorders in mice and rats. Folia Pharmacol. Japon. 71, 195-210 (1975) (Abs. in English)

2 Hata, T., Kita, T., Itoh, E. and Harada, N.: Experimental studies on optimal conditions of loading SART stress (repeated cold stress) upon animals. Japan. J. Psychosom. Med. 24, 257266 (1984)

3 Uchida, S., Takeyasu, K., Noguchi, Y., Yoshida, H., Hata, T. and Kita, T.: Decrease in muscarinic acetylcholine receptors in the small intestine of mice subjected to repeated cold stress. Life Sci. 22, 2197-2203 (1978)

4 Kita, T., Hata, T., lida, J., Yoneda, R. and Ishida, S.: Decrease in pain threshold in SART stressed mice. Japan. J. Pharmacol. 29, 479-482 (1979)

5 Hata, T., Kita, T., Namimatsu, A., Itoh, E, and Oda, $Y .:$ Changes of blood pressure and regional blood flow in SART rats and drug actions on these changes. Folia Pharmacol. Japon. 79, 335-342 (1982) (Abs. in English)

6 Hata, T., Kita, T., Itoh, E. and Namimatsu, A.: Changes of the function in the heart of SART stressed (repeated cold stressed) mice and the action of Neurotropin on these changes. Folia Pharmacol. Japon. 79, 487-492 (1982) (Abs. in English)

7 Hata, T., Nishida, S., Kita, T., Itoh, E. and Harada, $\mathrm{N}$.: Change of the immune response in SART stressed animals and influences of some drugs on it. Shinshin-Igaku 24, 301-308 (1984) (Abs. in English)

8 Kita, T., Hata, T., Itoh, E. and Harada, N.: Methacholine- and adrenaline-induced arrhythmias in repeatedly cold-stressed mice. Japan. J. Pharmacol. 35, 327-329 (1984)

9 Hata, T., Kita, T., Kawabata, A., Itoh. E. and Nishimura, Y.: Changes of tissue blood flow in mice loaded with SART (repeated cold) stress or restraint and water immersion stress and the effect of administered Neurotropin. Japan. J. Pharmacol. 41, 69-79 (1986)

10 Kita, T., Hata, T. Higashiguchi, T., Itoh. E. and Kawabata, A.: Changes of total acetylcholine content and the activity of related enzymes in SART (repeated cold)-stressed rat brain and duodenum. Japan. J. Pharmacol. 40, 174-177 (1986)

11 Hata, T., Kita, T., Higashiguchi, T. and Ichida, S.: 
Total acetylcholine content, and activities of choline acetyltransferase and acetylcholinesterase in brain and duodenum of SART-stressed (repeated cold-stressed) rat. Japan. J. Pharmacol. 41, 475-485 (1986)

12 Hata, T., Kamanaka, Y., Kita, T., Honda, S., Itoh, E. and Kawabata, A.: Effects of Neurotropin and other drugs on changes in brain and plasma catecholamine content in SART-stressed rats. Japan. J. Pharmacol. 43, Supp. 153P (1987)

13 Hata, T., Kita, T., Kamanaka, Y., Honda. S., Kakehi, K., Kawabata, A. and Itoh, E.: Catecholamine levels in the brain of SART (repeated cold)-stressed rats. J. Auton. Pharmacol. 7. (1987) (in press)

14 Kita, T., Hata, T., Itoh, E. and Namimatsu, A.: Testing methods for vegetative syndrome in the rat and effects of Neurotropin and other drugs. Japan. J. Psychosom. Med. 23, 61-68 (1983)

15 Paxinos, G. and Watson, C.: The Rat Brain in Stereotaxic Coordinates. Academic Press Japan. Inc. Tokyo (1982)

16 Yamamoto, K.: Neurohormonal mechanisms relating to circadian sleep-wakefulness cycles of experimental animals. Shinkei-Shinpo 19, $771-$ 778 (1975) (Abs. in English)

17 Inoue, T.: Methods for EEG analysis. In Electroencephalography, Edited by Kadobayashi, I., Inoue. T. and Nakamura, M., p. 248-261. Kinpodo. Kyoto (1983) (in Japanese)

18 Young, G.A., Steinfels, G.F., Khazan, N. and Glaser, E.M.: Cartical EEG power spectra associated with sleep-awake behavior in the rat. Pharmacol. Biochem. Behav. 8, 89-91 (1978)

19 Wauquier, A., Niemegeers, C.J.E. and Jassen, P.A.J.: Neuropharmacological comparison between domperidone and metoclopramide. Japan. J. Pharmacol. 31, 305-314 (1981)

20 Barzaghi, F., Dragonetti, M., Formento, M.L. and Boissier, J.R.: Cerebral energy metabolism and computerized EEG. J. Pharmacol. 13, 553563 (1982)

21 Bronzino, J.D., Austin, K., Siok, C.J., Cordova, C. and Morgane, P.J.: Spectral analysis of neocortical and hippocampal EEG in the protein malnourished rat. Electroencephalogr. Clin. Neurophysiol. 55, 699-709 (1983)

22 Wolf, D.L., Young, G.A. and Khazan, N.: Comparison between ethanol-induced and slowwave sleep synchronous EFG activity utilizing spectral analysis. Neuropharmacology 20, 687692 (1981)

23 Depoortere, H., Decobert, M. and Honoré, L.: Drug effects on the EEG of various species of laboratory animals. Neuropsychobiology 9, 244249 (1983)

24 Yamamoto, J.: Characteristics of the cortical and hippocampal EEG power spectra of rabbits during normal behavioral states and after administration of CNS acting drugs. Japan. J. Pharmacol. 37, 227-234 (1985)

25 I wata, N. and Mikuni, N.: EEG change in the conscious rat during immobitity induced by psychological stress. Psychopharmacology (Berlin) 71, 117-122 (1980)

26 Yamaoka, S.: Relationship between brown adipose tissue and the brain activity I. EEG activity. J. Physiol. Soc. Japan. 31, 127-154 (1968) (Abs. in English)

27 Harada, M., Ishii, T. and Yano, S.: Changes in electroencephalograms of rats during the development of stress ulcer. Chem. Pharm. Bull. (Tokyo) 26, 746-751 (1978)

28 Amiragova, M.G.: Neurophysiological analysis of the development of endocrine and hypertensive reactions in prolonged emotional stress. Brain Res. 344, 303-315 (1985)

29 Nakano, K: EEG in patients with psychosomatic disorders. Igaku to Yakugaku 12, 1683-1686 (1.984) (in Japanese) 